\title{
CHAMBER MOTION MEASUREMENTS AT THE NSLS X-RAY RING*
}

\author{
L. Solomon", D. Lynch, BNL; J. Safranek, SSRL; O. Singh, ANL
}

\begin{abstract}
Work has been ongoing at the NSLS to improve the orbit stability of the X-Ray Ring by accounting for the thermal motion of the vacuum chamber, which supports the electron beam position monitors (BPMs). In-situ contact measurements of the vacuum chamber motion have been carried out using support stands that have been designed and extensively tested to reduce errors associated with thermal changes in the stands themselves. Using this chamber motion as a correction to the orbit motion measured by the BPMs, the precise location of the radiation beam can be predicted. These predictions are compared with actual radiation beam measurements on the experimental floor, and with predictions based solely on BPM measurements of the electron beam position. This paper reviews this work including stand design and performance, chamber motion measurements, predictions based on these data, and results.
\end{abstract}

\section{INTRODUCTION}

In order to stabilize the electron beam position in the NSLS X-Ray Ring, pickup electrodes monitor the beam position and are used in the feedback system for stabilization. However, the temperature of the vacuum chamber is a function of time due to radiative heating, and therefore its motion is also a function of time. Thus, in order to stabilize the motion in a stationary coordinate system it is necessary that both the beam motion with respect to the chamber, and the chamber motion itself, are monitored and used in the feedback. Towards this end a) motion of the vacuum chamber has been measured and correlated to the motion of the radiation on the X-Ray Ring floor, and b) carbon fiber stands which are extremely stable and relatively insensitive to temperature changes within the ring have been designed and tested. This paper summarizes the results of this work to date.

\section{BEAM MOTION}

The motion of the beam at the source point of the X28 beamline was measured with a pinhole camera. The

\footnotetext{
Work performed under the auspices of the U.S.Department of Energy

"Email: solomon@bnl.gov
}

beam position with respect to the vacuum chamber on two BPMs just upstream of X28 (BPM 33 and 34) and the first BPM downstream (BPM 35) was also measured, along with the horizontal vacuum chamber motion at the three BPM locations. For measurement of the vacuum chamber motion, linear voltage displacement transducers are mounted on stands and are used to monitor the horizontal motion. These transducers have a range of $+/$ 0.125 inches, and with our readback electronics the digital resolution is 0.4 microns/count. Each of the LVDT devices was calibrated individually throughout its range of motion, with its cabling and circuitry.

The shift in the electron beam orbit at the $\mathrm{X} 28$ source point can be predicted given the measured change in beam position at nearby BPMs. This assumes that the orbit shift is caused by magnetic field changes elsewhere in the ring. The magnetic field changes between the BPMs and the source point must be small.

Three BPMs are needed to accurately predict horizontal motion at the source point, because horizontal orbit motion can be caused by a change in electron energy $(\delta)$ as well as in horizontal betatron phase space $\left(x, x^{\prime}\right)$. A significant fraction of the closed orbit shift over the course of a fill in the X-Ray Ring is associated with $\delta$ [1]. Given the $3 \times 3$ transfer matrices for $x, x^{\prime}$ and $\delta$ and the measured orbit shifts at the three BPMs, the orbit motion at X28 can be predicted.

Through the course of a 12 hour fill of the X-Ray Ring, the horizontal motion of the vacuum chamber at BPM 33, 34 and 35 location was about 30,130, and 160 microns respectively. By comparing the chamber motion data with the BPM data, it is seen that the chamber motion is reflected in an apparent motion of the electron beam to varying degrees for the various locations. For example, at BPM 34 location, almost all of the electron beam motion is due to chamber motion.

In Figure 1, the measured horizontal motion of the radiation beam at the source point is compared with predictions based solely on the motion of the electron beam, and also on the movement of both the chamber and the electron beam with respect to the chamber. The prediction incorporating the motion of the vacuum chamber closely mimics the actual radiation beam motion. These results provide a strong impetus towards real-time measurement and incorporation of the vacuum chamber motion into the feedback as a means towards a significantly more stable beam. 
Stand A vs Stand B Motion

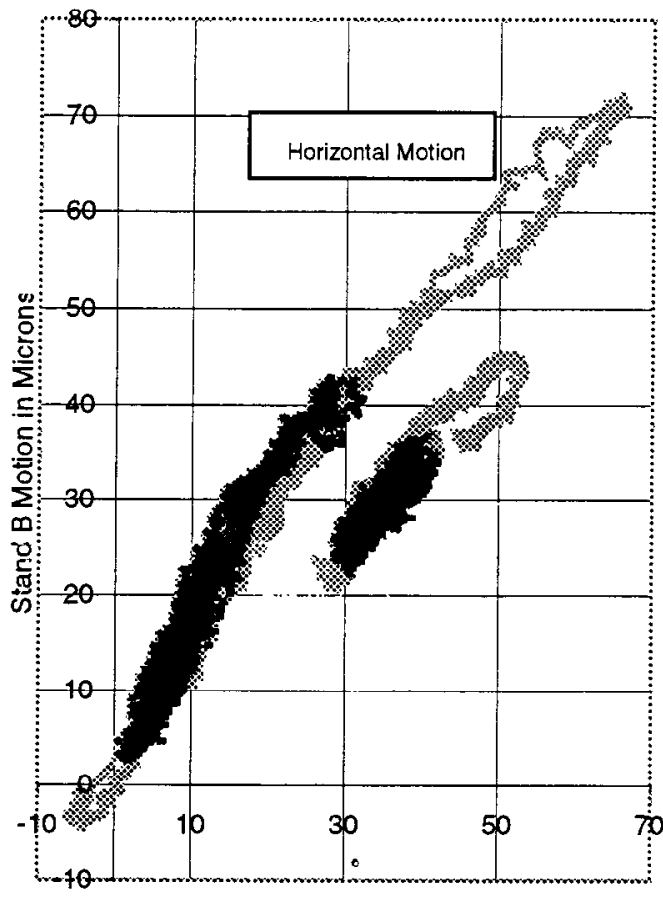

Stand A Motion in Microns

Figure 2 The horizontal and vertical motion recorded by one carbon fiber test stand compared with another carbon test stand. The upper data is the horizontal motion, and the lower data is the vertical motion. The lighter grey data was collected over an 11 day period, during which there was a $7^{\circ}$ air temperature change, and a $4.8^{\circ}$ temperature change in the granite. The darker data points represent 6.5 days of data, with a $5.6^{\circ}$ air temperature change and a $2.8^{\circ}$ temperature change in the granite.

Because of this large temperature effect associated with the granite table, the behavior of the two test stand motions were compared to each other to measure the stability of the stands as a function of temperature and time. Any undesirable behavior in the stability of the stands should not be simultaneously seen in both of the two tested stands. As can be seen in Figure 2, over the course of an 11 day period, the two stands tracked each other in both the horizontal and the vertical directions within $+/-5$ microns. This level of stability over such a long term (11 days) is excellent. Actually, the relevant time scale for actual use of these stands is the twelve hour fill period of the X-Ray Ring. Over this time, the stands track each other to within a couple of microns. Given these test results, current plans are to re-measure both vertical and horizontal ring movements at several locations with these improved stands, and test incorporation of this data into the feedback system during study periods at NSLS.

\section{SUMMARY}

In summary, measurements have been performed which indicate that significant improvement in the radiation beam stability can be expected when the motion of the vacuum chamber is accounted for. This is seen through comparison of beam motion predictions bascd on strictly BPM measurements, and on BPM and vacuum chamber motion measurements. Thus, very accurate chamber motion measurements are required. Stable support stands have been built and tested, with long term stability results on the order of several microns. Presently, measurement of the chamber motion with these improved stands, and utilization of the data in the orbit feedback system is being implemented in certain areas within the X-Ray Ring at NSLS.

\section{ACKNOWLEDGEMENTS}

The authors would like to thank M. Lehecka for his technical assistance throughout the course of this work, and S. Krinsky for guidance. This work performed under the auspices of the U.S. Department of Energy under contract DE-AC02-98CH10886

\section{REFERENCES}

[1] J. Safranek, O. Singh, and L. Solomon, "Orbit Stability Improvement at the NSLS X-Ray Ring", 1995 Particle Accelerator Conference, Dallas, Texas. 